\title{
Origin of Diffuse X-ray Emission and Ionization in the GC
}

\author{
V. Dogiel ${ }^{* 1,2,3}$; D. Chernyshov ${ }^{1,2}$, V. Tatischeff ${ }^{4}$, A. Kiselev ${ }^{1}$, K. S. Cheng ${ }^{2}$, C. K. \\ Leung $^{2}$, and R. Terrier ${ }^{5}$ \\ ${ }^{1}$ I.E.Tamm Theoretical Physics Division of P.N.Lebedev Institute of Physics, Leninskii pr, 53, \\ 119991 Moscow, Russia \\ ${ }^{2}$ Department of Physics, University of Hong Kong, Pokfulam Road, Hong Kong, China \\ ${ }^{3}$ Moscow Institute of Physics and Technology (State University), 9, Institutsky lane, \\ Dolgoprudny, 141707, Russia \\ ${ }^{4}$ Centre de Spectrométrie Nucléaire et de Spectrométrie de Masse, IN2P3/CNRS and Univ \\ Paris-Sud, 91405 Orsay Campus, France \\ ${ }^{5}$ Astroparticule et Cosmologie, Université Paris7/CNRS/CEA, Batiment Condorcet, 75013 Paris, \\ France
}

Unlike the time variable iron line emission from molecular clouds which is produced by past activity of $\operatorname{Sgr} A^{*}$, the origin of the diffuse line emission in the Galactic center (GC) region is debated. We investigated the origin of this line emission and a source of $\mathrm{H}_{2}$ ionization within $200 \mathrm{pc}$ central radius. We showed that iron atoms and $\mathrm{H}_{2}$ molecules in the diffuse interstellar medium of the GC are not ionized by the same particles. Iron atoms of the diffuse gas are most likely ionized by X-ray photons emitted by Sgr A* while the molecular hydrogen in the GC region is likely ionized by cosmic rays. Production of the line emission from molecular clouds by cosmic rays (CRs) depends strongly on processes of CR penetration into them. We show that turbulent motions of neutral gas may generate strong magnetic fluctuations in the clouds, which prevent free penetration of CRs into the clouds from outside. We provided also analysis of $\mathrm{H}_{2}$ ionization within $1 \mathrm{pc}$ (CND) central region and showed that the ionization there is likely provided by subrelativistic CRs.

10th INTEGRAL Workshop: "A Synergistic View of the High Energy Sky" - Integral2014, 15-19 September 2014 Annapolis, MD, USA

\footnotetext{
* Speaker.

†E-mail: dogiel@td.lpi.ru
} 


\section{Introduction}

The distribution of molecular hydrogen in the Galactic central (CG) region $(R \leq 100 \mathrm{pc})$ is strongly nonunform. Most of the gas is concentrated in dense $\left(n_{H} \geq 10^{4} \mathrm{~cm}^{-3}\right)$ giant molecular clouds whose mass is about $M \leq 10^{6} M_{\odot}$ (see the reviews [[, []]).

Some of the GC molecular clouds are seen in the $6.4 \mathrm{keV}$ iron line which is excited when the $\mathrm{K}$ shell of the iron atom is ionized. The origin this line is nonthermal because the temperature of the clouds is about $100 \mathrm{~K}$. Up to recent time different interpretations of ionization were suggested but the discovery of temporal variations of the line emission with a period about 10 years (see

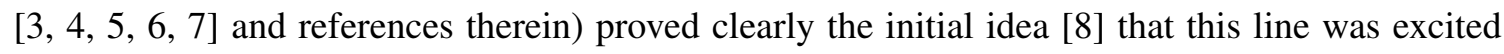
by a flux of hard X-rays emitted about 100 years ago by a central source, probably by the central black hole Sgr A*. The required X-ray luminosity in this case is about $10^{39} \mathrm{erg} \mathrm{s}^{-1}$. Indeed, the expected time variations of the iron line in the case of its photon origin is about 10 years, for which the front of $\mathrm{X}$-rays crosses a cloud of several pc radius.

Recently a new diffuse component of hydrogen was discovered (see [Q, 四]), which fills more or less uniformly the region of the $200 \mathrm{pc}$ radius around the GC with the density $n \sim 10^{2} \mathrm{~cm}^{-3}$. This hydrogen may emit a truly diffuse $6.4 \mathrm{keV}$ emission found by Suzaku [W]. This emission is concentrated in an extended region in the GC, with angular scales $\ell \sim 0.6^{\circ}$ in longitude and $b \sim 0.2^{\circ}-0.4^{\circ}$ in latitude. According to [W] this diffuse component of the $6.4 \mathrm{keV}$ line may have completely different origin than that from the clouds.

Another processes of ionization was found from measurements of the IR absorption lines generated by ionizedf molecular hydrogen in the GC. These measurements found the ionization rate in the GC about $\zeta \sim(1-3) \times 10^{-15} \mathrm{~s}^{-1}$, which is two orders of magnitude higher than in other parts of the Galaxy (see [미 and references therein). The source of ionization is still debated.

Below we discuss ionization processes of iron and hydrogen in the molecular clouds and in the diffuse component of hydrogen.

\section{Ionization of the GC Molecular Clouds by CRs}

Most of the GC molecular clouds show temporal variations of the $6.4 \mathrm{keV}$ line that is in favour of iron ionization by hard X-rays emitted by Sgr A*. It could be another component of the line flux from the clouds, which is produced by CRs if their density inside the cloud is high enough. Gamma-ray observations showed that CRs of high energies penetrated into the cloud [피, 때, ㅁ5]. Moreover, there are indications that in some molecular complexes iron atoms mainly are ionized by CRs (see [1]6]).

One of the key problem of interaction between CRs and molecular clouds is, how CRs penetrate into the dense hydrogen gas of the clouds. This problem in nontrivial.

It is known that propagation of charged particles in the interstellar medium is determined by particle scattering on magnetic field fluctuations . The frequency of resonant scattering on MHDwaves is described (see e.g. [피]) as

$$
v \sim 2 \pi^{2} \omega_{H} \frac{k W(k)}{H^{2}}
$$


where $H$ is the large scale magnetic field, $k$ is the wave number of fluctuations, for the resonat scattering $k \sim \mathrm{ZeH} / p c \mu$ (here $p$ is the particle mometum and $\mu$ is the particle pitch-angle), $W(k)$ is the spectrum of magnetic fluctuations, and $\omega_{H}=e H / m c$. For high enough scattering frequency particle propagation can be described as diffusion with the effective diffusion coefficient

$$
D \sim \frac{v^{2}}{v}
$$

where $\mathrm{v}$ is the particle velocity.

In a low ionized gas of molecular clouds MHD-waves are damped due to ion-neutral friction (see [[18]), i.e. particle propagate there without scattering. On the other hand, the neutral gas in the molecular clouds is strongly turbulized (see the review [ㅁ]). These turbulent motions excite fluctuations of magnetic field. As a result a spagetti like structure of magnetic field line is generated inside the clouds and the energy of magnetic fluctuations is concentrated at the correlation length of magnetic field, $L$, which is much smaller than a size of the molecular cloud [201, 2]]. Propagation of magnetized charged particles along tangled magnetic field lines can also be descibed as diffusion with the effective diffusion coefficient

$$
D \sim \mathrm{v} L
$$

which is constant for relativistic particles. In order to calculate the rate on ionzation by CRs we should define their spectrum inside the clouds.

In the dense gas of clouds CRs loose their energy by ionization with the rate

$$
\left(\frac{d E}{d t}\right)_{i} \equiv b(E)=-\frac{2 \pi n e^{4}}{m c \beta(E)} \ln \Lambda(E) .
$$

where $m$ is the electron mass, $\mathrm{v}$ is the proton velocity, $\beta=\mathrm{v} / c, \ln \Lambda$ is the Coulomb logarithm.

The spectrum of CRs inside the cloud, $N_{p}(E, x)$, can be derived from the equation (see [D2])

$$
\frac{\partial}{\partial E}(b(E) N)-D \frac{\partial^{2}}{\partial x^{2}} N=0
$$

where $x$ is the coordinate from the surface to the center.

The boundary conditions on the cloud surface $(x=0)$ is

$$
\left.N\right|_{x=0}=N_{0}(E)
$$

where $N_{0}$ is the CR spectrum in the intercloud medium.

With the derived spectrum $N(E)$ one can calculate the inization rate of hydrogen, $\zeta$, and the intensity of $6.4 \mathrm{keV}$ line emission, $F_{6.4}$, from the equations

$$
\begin{aligned}
& \zeta \simeq \int_{I\left(\mathrm{H}_{2}\right)}^{E^{\max }} d E \sigma_{p}^{\mathrm{ioni}}(E) N(E) \mathrm{v}(E) \\
& F_{6.4} \simeq \eta_{F e} n_{H} V \int_{I(\mathrm{Fe})}^{E^{\max }} d E \sigma_{p}^{\mathrm{Fe}}(E) N(E) \mathrm{v}(E)
\end{aligned}
$$


where $\sigma_{p}^{\text {ioni }}$ and $\sigma_{p}^{\mathrm{Fe}}$ are the cross-sections of hydrogen and iron ionization by protons taken e.g. from [23], $I\left(\mathrm{H}_{2}\right)$ is the ionization potentials of hydrogen, $I(\mathrm{Fe} \mathrm{K})=7.1 \mathrm{keV}$ is the ionization potentials of iron, $E^{\max }$ is the maximum energy of CRs, $\eta_{F e}$ is the iron abundance, and $V$ is the cloud volume.

Simple estimations [22] show that if the ionization rate in Sgr B2 is $4 \times 10^{-16} \mathrm{~s}^{-1}$ as measured in the GC (see [24]) then the expected $6.4 \mathrm{keV}$ flux produced by CR protons equals or is below $1 \%$ of the flux produced by X-ray photons from Sgr A*.

Our calculations showed also that hydrogen ionization in the GC by electrons (see e.g. [25, 26] is problematic because the density of electrons is strongly depressed by ionization losses in the intercloud medium. In our opinion, in spite of their short lifetime electrons are unable to reproduce the simultaneous short time variability of the iron line fluxes from the GC clouds which are distant from each other by hundred pc.

\section{Hydrogen Ionization in the CND}

One of the most massive molecular complexes with the radius of $R_{c}=3.5 \mathrm{pc}$, the circumnuclear disk (CND) is located directly in the GC and surrounds the central black hole, Sgr A*. Total mass of the CND was estimated between $(2-10) \times 10^{5} M_{\odot}[$ [Z, [2]].

Below we analyse processes of ionization in the CND which may differ from these processes in other molecular clouds (see [[28]). Observations of the Fermi LAT gamma ray telescope [ㅁ] found a prominent gamma-ray flux in the GeV region (the source 2FGL J1745.6-2858 in the second Fermi LAT source catalog [30]) that indicated that this region is filled with high energy cosmic rays (CRs). The ionization rate measured in the direction of this cloud from the IR data gave the value $\sim 1.2 \times 10^{-15} \mathrm{~s}^{-1}$, that does no differ from the average in the GC. However, the ionization rate calculated for the CR density in the CND derived from the gamma ray data was in four orders of magnitude higher (see [B]]). This discrepancy between estimations of the ionization rate from the IR and gamma ray data requires a special analysis, using kinetic equaions which describe CR propagation in the CND region.

Processes of hydrogen ionization in the CND was analysed in [28]. The estimated gamma-ray flux from this region (the source 2FGL J1745.6-2858) for $E>2 \mathrm{GeV}$ is about $I_{o b s}=1.08 \times 10^{-10}$ erg cm ${ }^{-2} \mathrm{~s}^{-1}$ with the spectral index $\gamma=2.68$ (see [29]). For the CND radius $R_{c}=3-5 \mathrm{pc}$ this gives the average gas density there of about $n_{H} \simeq 4 \times 10^{5} \mathrm{~cm}^{-3}$.

To estimate the spectrum of CR protons in this region we take the kinetic equation,

$$
\frac{\partial N}{\partial t}-\nabla(D \nabla N)+\frac{\partial}{\partial E}\left(\frac{d E}{d t} N\right)+\frac{N}{T}=Q(E, r, t)
$$

where $N(E)$ is the CR density, $E$ is the particle energy, $d E / d t$ is the rate of energy losses for protons or electrons, $Q(E)$ is the source function, $T$ is the particle lifetime, which e.g. is the time of $p-p$ collisions, and $D$ is an effective diffusion coefficient due to scattering on magnetic fluctuations.

If a point-like source ( $\left.\operatorname{Sgr} \mathrm{A}^{*}\right)$ emits a power-law momentum spectrum of particles $\left(\propto p^{-\gamma}\right)$ with the spectral index $\gamma$ then its energy spectrum can be presented as

$$
Q(r, E, t)=A(E, t) \delta(\mathbf{r})
$$


with

$$
A(E)=A_{0}(t) \frac{E+M c^{2}}{\left(E^{2}+2 M c^{2} E\right)^{(\gamma+1) / 2}}
$$

Here $M$ is a particle mass, $E$ its energy, and $A_{0}$ is proportional to the source power.

For the observed gamma ray flux from the CND we can estimate the spectrum of protons from the equation

$$
F_{\gamma}\left(E_{\gamma}, t\right)=\frac{n_{H} c}{R_{\odot}^{2}} \int_{0}^{R} r^{2} d r \int_{E} N(E, r, t) \frac{d \sigma}{d E_{\gamma}}\left(E, E_{\gamma}\right) d E,
$$

where $n_{H}$ is the average hydrogen density in the gamma-ray emitting region, $R_{\odot}=8 \mathrm{kpc}$ is the distance from Earth to the CND, and $d \sigma / d E_{\gamma}\left(E, E_{\gamma}\right)$ is the differential cross-section for gammaray production in proton-proton collisions [B2].

If the source stationary emits $\mathrm{CRs}$, the ionization rate of hydrogen produced by these protons in the CND is about $4.6 \times 10^{-15} \mathrm{~s}^{-1}$. So, unlike [B]], we do not see any descrepancy between the observed gamma-ray flux from the CND and the ionization rate there derived from the IR data. The estimated radio flux from the CND at $v=1.5 \mathrm{GHz}$ produced by secondary electrons is about 4.63 Jy.

We calculated also an X-rays flux generated by bremsstrahlung of protons and electrons in the range $20-40 \mathrm{keV}$ which is about $3 \times 10^{-13} \mathrm{erg} \mathrm{cm}^{-2} \mathrm{~s}^{-1}$. The spectral index of bremsstrahlung radiation in this range is -1.53 .

For the transient source of protons in the SgrA* of the form

$$
Q(E, r, t)=A(E) \delta(\mathbf{r}) \delta(t)
$$

the spectra of protons in relativistic and subrelativistic energy ranges are respectively

$$
\begin{gathered}
N^{r e l}(E, r, t)=\frac{A_{0} E^{-\gamma}}{(4 \pi D t)^{3 / 2}} e^{-t / \tau_{p p}} \exp \left(-\frac{r^{2}}{4 D t}\right) \\
N^{\text {subrel }}(E, r, t)=\frac{A_{0}^{\prime} \sqrt{E}}{(4 \pi D t)^{3 / 2}} \exp \left(-\frac{r^{2}}{4 D t}\right)\left(a t+E^{3 / 2}\right)^{-(\gamma+1) / 3}
\end{gathered}
$$

where the energy of ionization losses for subrelativistic protons is presented as

$$
\left(\frac{d E}{d t}\right)_{i}=-\frac{2 \pi e^{4} n_{H}}{m_{e} c \beta(E)} \ln \left(\frac{m_{e}^{2} c^{2} W_{\max }}{4 \pi e^{2} \hbar^{2} n}\right) \simeq \frac{a}{\sqrt{E}}
$$

Our calculations showed that the necessary ionization rate $1.2 \times 10^{-15} \mathrm{~s}^{-1}$ and the gamma-ray flux $I_{o b s}=1.08 \times 10^{-10} \mathrm{erg} \mathrm{cm}^{-2} \mathrm{~s}^{-1}$ can be obtained e.g. for the diffusion coefficient in the CND $D=3 \times 10^{27} \mathrm{~cm}^{2} \mathrm{~s}^{-1}$ if the proton ejection occured about $100 \mathrm{yr}$ ago.

In this case the radio flux produced by the secondary electrons is about $5 \mathrm{Jy}$ at $v=1.5 \mathrm{GHz}$ and the flux of hard X-rays in the range $20-40 \mathrm{keV}$ is about $1.3 \times 10^{-13} \mathrm{erg} \mathrm{cm}^{2} \mathrm{~s}^{-1}$. It is interesting to notice that the region of X-ray emission is more extended than the CND. The reason is that the lifetime of electrons emitting at the frequency $1.5 \mathrm{GHz}$ is quite long, about $10^{4} \mathrm{yr}$. Therefore, a significant fraction of these electrons, generated at initial stages, escape from the CND and form a halo around this region. They generate X-ray emission in the halo by inverse Compton while this emission in the CND is produced by bremsstrahlung. 


\section{Ionization of the Diffuse Molecular Gas in the GC}

As we mentioned in the Introduction the origin of the $\mathrm{Fe} I \mathrm{~K} \alpha$ line emission from the diffuse gas in the GC might be different from that of the dense clouds, i.e. it may be generated by sources other than the hard X-ray photons from Sgr A*. The origin of the diffuse component cannot be determined from time variability as in the case of compact clouds. Unlike the line emission from the clouds, the diffuse flux of $6.4 \mathrm{keV}$ line generated by Sgr A* photons is almost constant for several hundred years, just for the time needed for a photon to cross the $100 \mathrm{pc}$ GC region (see [B3]]). Therefore other selection criteria should be developed.

One of the questions is whether the ionization of diffuse iron and hydrogen in the GC have the same origin, i.e. whether they are ionized by the same particles: photons or by CRs or not. This problem was analysed in [34].

If ionization of hydrogen and iron is produced by CRs, it is easy to calculate the ratio of their ionization rates, $X_{6.4, i}$, which is

$$
X_{6.4, i}^{e s t} \approx \frac{\eta_{\mathrm{Fe}}}{f_{\mathrm{H}_{2}}} \frac{\int_{I(\mathrm{Fe} \mathrm{K})}^{E_{\max }} N_{i}(E) \sigma_{i \mathrm{Fe}}^{\mathrm{K} \alpha}(E) v_{i}(E) d E}{\int_{I\left(\mathrm{H}_{2}\right)}^{E_{\max }} N_{i}(E) \sigma_{i \mathrm{H}_{2}}^{\text {ioni }}(E) v_{i}(E) d E},
$$

where $I(\mathrm{Fe} \mathrm{K})=7.1 \mathrm{keV}$ and $I\left(\mathrm{H}_{2}\right)=15.6 \mathrm{eV}$ are the ionization potentials of the $\mathrm{K}$ shell of $\mathrm{Fe}$ and of $\mathrm{H}_{2}$, respectively, $\eta_{\mathrm{Fe}}$ is the Fe abundance, $f_{\mathrm{H}_{2}}=n\left(\mathrm{H}_{2}\right) /\left(2 n\left(\mathrm{H}_{2}\right)+n(\mathrm{H})\right)$ the fractional density of $\mathrm{H}_{2}$ molecules relative to the total number of $\mathrm{H}$ atoms, $N_{i}(E)$ the differential equilibrium number of CRs of type $i$ propagating in the diffuse gas, $v_{i}(E)$ the velocity of these particles, $E_{\max }$ their maximum kinetic energy, $\sigma_{i \mathrm{Fe}}^{\mathrm{K} \alpha}(E)$ the cross section for producing the $6.4 \mathrm{keV}$ line by interaction of fast particles of type $i$ with Fe atoms, and $\sigma_{i \mathrm{H}_{2}}^{\text {ioni }}(E)$ the cross section for ionization of the $\mathrm{H}_{2}$ molecule by the particle $i$. We have neglected in this equation the contribution of secondary electrons for both $6.4 \mathrm{keV}$ line production and $\mathrm{H}_{2}$ ionization.

As follows from calculations of [B4] the value of $X_{6.4, i}^{\text {est }}$ is less than $7 \times 10^{-8}$ in units: $6.4 \mathrm{keV}$ photons per one $\mathrm{H}_{2}$ ionization.



$$
X_{6.4, i}^{o b s} \simeq(0.6-1.9) \times 10^{-6},
$$

i.e, more than order of magnitude higher than the estimation $X_{6.4, i}^{\text {est }}$. Thus, CRs are unable to ionize simultaneously iron and hydrogen in the GC.

The diffuse hydrogen can be effectively ionized by low energy cosmic rays (electron or protons, see [ㄷ] and [34]) but as it follows from Eqs. (4.]) and (4.2), CRs are unable to provide enough ionization of iron there. The CR luminosity requred for the hydrogen ionization $3 \times 10^{-15}$ $\mathrm{s}^{-1}$ is about $2 \times 10^{39} \mathrm{erg} \mathrm{s}^{-1}$ that can be supplied by energy sources in the GC (see [B]]) .

With appropriate equations we can investigate the alternative process, ionization of hydrogen and iron by Sgr A* photons. The distribution of primary photons in the disk as a function of energy $E_{X}$ and radius $r$ is then given by

$$
n_{\mathrm{ph}}\left(E_{X}, r\right)=\frac{Q_{0}}{4 \pi c r^{2} E_{X}^{2}} \exp \left(-\left\langle n_{\mathrm{H}_{2}}\right\rangle \sigma_{\mathrm{abs}}\left(E_{X}\right) r / f_{\mathrm{H}_{2}}\right) \theta(r-c T) \theta(c T+c \Delta t-r),
$$


where $\theta(x)$ is the Heaviside function, $\sigma_{\text {abs }}$ the photoelectric absorption cross section per $\mathrm{H}$ atom, $\Delta t$ is the duration of the $\mathrm{X}$-ray flare, $T$ is the time passed from flare termination, and $n_{\mathrm{H}_{2}}$ is the hydrogen density in the GC. The flux of primary X-ray photons from Sgr A* was taken in the form $Q_{0} E_{x}^{-2}$ (see [B]] and [3]]).

The longitudinal distribution of $6.4 \mathrm{keV}$ line emission in the direction $\mathbf{I}$ can be calculated from

$$
\left.I_{6.4}(\ell)=\frac{\eta_{\mathrm{Fe}}\left\langle n_{\mathrm{H}_{2}}\right\rangle}{4 \pi f_{\mathrm{H}_{2}}} \int_{I(\mathrm{Fe} \mathrm{K})}^{E_{\mathrm{max}}} \sigma_{X \mathrm{Fe}}^{\mathrm{K} \alpha}\left(E_{X}\right) \int_{\mathbf{l}} n_{\mathrm{ph}}\left(E_{X}, r\right) d \mathbf{l}\right) d E_{X},
$$

where $\sigma_{X \mathrm{Fe}}^{\mathrm{K} \alpha}$ is the cross section for producing the $6.4 \mathrm{keV}$ line by Fe K-shell photoionization [[23].

This equation desribes nicely the spatial distribution and intensity of the diffuse $6.4 \mathrm{keV}$ line in the GC. The necessary X-ray luminosity of Sgr A* is $\sim 10^{39} \mathrm{erg} \mathrm{s}^{-1}$ if $\Delta t=10 \mathrm{yr}$ and $\sim 10^{38}$ $\operatorname{erg~s}^{-1}$ if $\Delta t=100 \mathrm{yr}$.

With these parameters of the primary X-ray flux we can calculate the ionization rate due to both photoelectric ionization and Compton scattering which is:

$$
\begin{aligned}
& \zeta_{2}(r, t) \simeq \int_{I\left(\mathrm{H}_{2}\right)}^{E_{\max }} d E_{X} \sigma_{X \mathrm{H}_{2}}^{\mathrm{ioni}}\left(E_{X}\right) c n_{\mathrm{ph}}\left(E_{X}, r, t\right) M_{\mathrm{sec}}\left(E_{X}\right)+ \\
& 2 \int_{E_{\mathrm{I}}}^{E_{\max }} d E_{X} c n_{\mathrm{ph}}\left(E_{X}, r, t\right) \int_{I\left(\mathrm{H}_{2}\right)}^{E_{e}^{\max }} d E_{e} \frac{d \sigma_{c}}{d E_{e}} M_{\mathrm{sec}}\left(E_{e}\right),
\end{aligned}
$$

where $\sigma_{X \mathrm{H}_{2}}^{\mathrm{ioni}}$ is the $\mathrm{H}_{2}$ photoelectric ionization cross section [38], $d \sigma_{c} / d E_{e}$ the Klein-Nishina differential cross-section as a function of the energy of the recoil electron $E_{e}, M_{\mathrm{sec}}\left(E_{e}\right)=\left[E_{e}-\right.$ $\left.I\left(\mathrm{H}_{2}\right)\right] / W$ with $W \approx 40 \mathrm{eV}$ [B]] the mean multiplicity of $\mathrm{H}_{2}$ ionization by a secondary electron, $E_{\mathrm{I}} \approx \sqrt{m_{e} c^{2} \mathrm{I}\left(\mathrm{H}_{2}\right) / 2}$ the minimum energy of an X-ray photon to ionize an $\mathrm{H}_{2}$ molecule by Compton scattering, $E_{e}^{\max }=2 E_{X}^{2} /\left(m_{e} c^{2}+2 E_{X}\right)$, and $n_{\mathrm{ph}}\left(E_{X}, r, t\right)$ is given by Eq. (4.3]).

It follows, however, from the numerical calculations of Eq. (4.5) (see [B4]) that the ionization rate of hydrogen produced by the primary photons is more than one order of magnitude less than observed while the generate enough $6.4 \mathrm{keV}$ photons. Then we conclude that the origin of $6.4 \mathrm{kev}$ line and hydrogen ionization is different, the first is produced by the X-ray photons from Sgr A* while the second by low energy cosmic rays.

\section{Conclusion}

Here is a summary of our analyis of ionization processes in the GC region.

- Efficiency of CR penetration into dense molecular clouds depends strongly on the structure of magnetic field inside the clouds. Two limit case are possible: 1 . when CRs freely propagate through the cloud without scattering if magnetic fluctuations are damped due to friction of ion and neutral components of the gas in the clouds; 2 . The observed turbulence of neutral gas in the clouds may excite fluctuations of the magnetic field. In some conditions the energy of magnetic fluctuations is concentrated at scales which are much smaller that the cloud size. Then, propagation of magnetized charged particles along tangled magnetic field lines can be described as diffusion with a relatively small diffusion coefficient; 
- When the front of primary photons emitted by Sgr A*, leaves a cloud, the intensity of the 6.4 $\mathrm{keV}$ line from there is determined by CRs. This situation is expected for the cloud Sgr B2, because as follows from observations the front of Sgr A* photons has crossed already the cloud. Our estimations for this cloud show that the background (stationary) flux from this cloud generated by CRs is about several per cents in the most favourable case. Otherwise, the flux of iron emission is negligible.

- We do not see any conflict between gamma-ray data and the rate of hydrogen ionization as it was mentioned in previous publications. For the proton spectrum, which we derived from the gamma-ray data, we obtained the ionization rate in the CND region about $10^{-15} \mathrm{~s}^{-1}$ (about the average value in the $\mathrm{GC}$ region) if this ionization is provided by $\mathrm{CR}$ protons;

- We estimated the spectrum of hard X-ray in the range $20-40 \mathrm{kev}$ produced by CRs in the CND region. This flux is about $10^{-13} \mathrm{erg} \mathrm{cm}^{-2} \mathrm{~s}^{-1}$. In the CND region, this flux is generated by inverse bremsstrahlung of protons and by bremsstrahlung of secondary electrons. If, however, a part of secondary electrons escapes from the CND region, then they generate by inverse Compton an additional X-ray flux in the medium surrounding the CND which can be seen as an X-ray halo around the CND.

- We showed that the ionization of iron in the diffuse hydrogen gas in the GC is also provided by primary X-ray photons emitted by Sgr A* hundred years ago, as in the case of the GC molecular clouds. Ionization of background hydrogen by these photons is negligible. We showed that hydrogen is ionized by low energy CR, most likely by protons;

\section{Acknowledgements}

VAD and DOC acknowledge support from the RFFI grants 15-52-52004, 15-02-02358, 1502-08143. DOC and AMK are supported in parts by the LPI Educational-Scientific Complex and Dynasty Foundation. KSC is supported by the GRF Grants of the Government of the Hong Kong SAR under HKU 701013. KSC, DOC, and VAD acknowledge support from the International Space Science Institute to the International Team "New Approach to Active Processes in Central Regions of Galaxies".

\section{References}

[1] Ferrière, K., Gillard, W., \& Jean, P. 2007, A\&A, 467, 611

[2] Ferrière, K. 2012, A\&A, 540, 50

[3] Inui, T., Koyama, K., Matsumoto, H., \& Tsuru T. Go 2009, PASJ, 61, 241

[4] Ponti, G., Terrier, R., Goldwurm, A. et al. 2010, ApJ, 714, 732

[5] Nobukawa, M., Ryu, S. G., Tsuru Go, T., \& Koyama, K. 2011, ApJ, 739, L52

[6] Clavel, M., Terrier, R., Goldwurm, A. et al. 2013, A\&A, 558, 32

[7] Clavel, M., Soldi, S., Terrier, R., et al. 2014, MNRAS, 443, L129

[8] Sunyaev, R. A., Markevitch, M., \& Pavlinsky, M. 1993, ApJ, 407, 606 
[9] McCall, B. J., Hinkle, K. H., Geballe, T. R. et al. 2002, ApJ, 567, 391

[10] Oka, T., Geballe, Th. R., Goto, M. et al. 2005, ApJ, 632, 882

[11] Uchiyama, H., Nobukawa, M., Tsuru Go, T., \& Koyama, K. 2013, PASJ 65, 19

[12] Geballe, T. R. 2012, Phil. Trans. R. Soc. A 370, 5151

[13] Aharonian, F. A. 2001, SSRv, 99, 187

[14] Hui, C. Y., Wu, E. M. H., Wu, J. H. K. et al. 2011, ApJ, 735, 115

[15] Yang, R., Jones, D. I., \& Aharonian, F. 2014, arXiv1410.7639

[16] Capelli, R., Warwick, R. S., Porquet, D., Gillessen, S., \& Predehl, P. 2012, A\&A, 545, A35

[17] Berezinsky, V.S., Bulanov, S.V., Dogiel, V.A., Ginzburg, V.L., \& Ptuskin, V.S.: 1990, Astrophysics of Cosmic Rays, (ed.V.L.Ginzburg), North Holland

[18] Kulsrud, R. \& Pearce, W. P. 1969, ApJ, 156, 445

[19] Hennebelle, P. \& Falgarone, E. 2012, A\&ARv, 20, 55

[20] Dogiel, V. A., Gurevich, A. V., Istomin, Ia. N., \& Zybin, K. P. 1987, MNRAS, 228, 843

[21] Istomin, Ya. N. \& Kiselev, A. M. 2013, MNRAS.436.2774I

[22] Dogiel, V. A., Chernyshov, D. O., Kiselev, A. M., \& Cheng, K. S. 2014, APh, 54, 33

[23] Tatischeff, V. 2003, EAS, 7, 79

[24] Van der Tak, F. F. S., Belloche, A., Schilke, P. et al. 2006, A\&A, 454, L99

[25] Yusef-Zadeh, F., Hewitt, J. W., Wardle, M. et al. 2013a, ApJ, 762, 33

[26] Yusef-Zadeh, F., Wardle, M., D. Lis, D. et al. 2013b, JPCA, 117, 9404

[27] Christopher M. H., Scoville, N. Z., Stolovy, S. R., \& Yun, Min S. 2005, ApJ, 622, 346

[28] Chernyshov, D. O., Leung, C. K., Cheng, K. S., Dogiel, V. A., \& Tatischeff, V. 2014, arXiv: 1412.2740

[29] Chernyakova, M., Malyshev, D., Aharonian, F. A. et al. 2011, ApJ, 726, 60

[30] Nolan, P. L., Abdo, A. A., Ackermann, M. et al. 2012, ApJS, 199, 31

[31] Goto, M., Indriolo, N., Geballe, T.R., Usuda, T. 2013, The Journal of Physical Chemistry A, 117, 9919

[32] Kamae, T., Karlsson, N., Mizuno, T., et al., 2006, ApJ, 647, 692

[33] Chernyshov, D., Dogiel, V., Nobukawa, M. et al. 2012, PASJ, 64, 14

[34] Dogiel, V. A., Chernyshov, D. O., Tatischeff, V., Cheng, K. S., \& Terrier, R. 2013, ApJ, 771, L43

[35] Crocker, R. M., Jones, D. I., Aharonian, F. et al. 2011, MNRAS, 413, 763

[36] Koyama, K., Takikawa, Y., Hyodo, Y. et al. 2009, PASJ, 61, S255

[37] Terrier, R., Ponti, G., Belanger, G. et al. 2010, ApJ, 719, 143

[38] Yan, M., Sadeghpour, H. R., \& Dalgarno, A. 2001, ApJ, 559, 1194

[39] Dalgarno, A., Yan, M., \& Liu, W.-H. 1999, ApJS, 125, 237 